This is a self-archived version of an original article. This version may differ from the original in pagination and typographic details.

Author(s): Lyyra, Pessi; Myllyneva, Aki; Hietanen, Jari K.

Title: Mentalizing eye contact with a face on a video : Gaze direction does not influence autonomic arousal

Year: 2018

Version: Accepted version (Final draft)

Copyright: (c) 2018 Scandinavian Psychological Associations and John Wiley \& Sons Ltd

Rights: In Copyright

Rights url: http://rightsstatements.org/page/InC/1.0/?language=en

Please cite the original version:

Lyyra, P., Myllyneva, A., \& Hietanen, J. K. (2018). Mentalizing eye contact with a face on a video

: Gaze direction does not influence autonomic arousal. Scandinavian Journal of Psychology, 59(4), 360-367. https://doi.org/10.1111/sjop.12452 
Running head: MENTALIZING EYE CONTACT

Mentalizing eye contact with a face on a video: Gaze direction does not influence autonomic arousal

Pessi Lyyra $^{1,2}$, Aki Myllyneva ${ }^{1}$, Jari K. Hietanen ${ }^{1}$

${ }^{1}$ Human Information Processing Laboratory, Faculty of Sciences/Psychology, FI-33014 University of Tampere, Tampere, Finland

${ }^{2}$ Department of Psychology, University of Jyväskylä, Jyväskylä, FI-40014

* Corresponding author, Human Information Processing Laboratory, School of Social Sciences and Humanities/Psychology, FI-33014 University of Tampere, Tampere, Finland

Email: pessi.lyyra@jyu.fi

Word count: 5,059 (excl. Abstract, references and figure legends) 
Running head: MENTALIZING EYE CONTACT

\begin{abstract}
Recent research has revealed enhanced autonomic and subjective responses to eye contact only when perceiving another live person. However, these enhanced responses to eye contact are abolished if the viewer believes that the other person is not able to see back to the viewer. We purported to investigate whether this "genuine" eye contact effect can be reproduced with prerecorded videos of stimulus persons. Autonomic responses, gaze behavior, and subjective selfassessments were measured while participants viewed pre-recorded video persons with direct or averted gaze, imagined that the video person was real, and mentalized that the person could see them or not. Pre-recorded videos did not evoke similar physiological or subjective eye contact effect as previously observed with live persons, not even when the participants were mentalizing of being seen by the person. Gaze tracking results showed, however, increased attention allocation to faces with direct gaze compared to averted gaze directions. The results suggest that elicitation of the physiological arousal in response to genuine eye contact seems to require spontaneous experience of seeing and of being seen by another individual.
\end{abstract}

Keywords: Eye contact; Mentalizing; Skin conductance response; Heart rate; Gaze tracking 
Running head: MENTALIZING EYE CONTACT

\section{Mentalizing eye contact with a face on a video: Gaze direction does not influence autonomic arousal}

Human social interaction is guided by a complex system of perceptual and higher-level cognitive processes. One essential function of this cognitive system is to analyse other individuals' gaze directions. Gaze carries information particularly about the direction of attention. Another person gazing towards oneself signals a potential approach or appraisal by that person and raises the need for a possible reaction. Perception of someone's eyes directed at oneself automatically triggers a specific set of cognitive and physiological responses (Senju \& Johnson, 2009). For example, direct gaze attracts visual attention (Böckler, van der Wel, \& Welsh, 2014; Conty, Tijus, Hugueville, Coelho, \& George, 2006; Doi, Ueda, \& Shikohara, 2009; Lyyra, Astikainen, \& Hietanen, 2017; Senju \& Hasegawa, 2005; von Grünau \& Anston, 1995; for a critical view of visual search studies, see Cooper, Law, \& Langton, 2013), and lengthens total viewing times of faces (Palanica \& Itier, 2012; Wieser, Pauli, Alpers, \& Mühlberger, 2009). Physiological measurements have shown that, compared to averted gaze, direct gaze induces greater heart rate deceleration responses indicative of attention orienting (Akechi et al., 2013; Myllyneva \& Hietanen, 2015a) and heightened autonomic sympathetic arousal reflecting preparation for action as measured by skin conductance responses (SCR) (Helminen, Kaasinen, \& Hietanen, 2011; Hietanen, Leppänen, Peltola, Linna-Aho, \& Ruuhiala, 2008; Nichols \& Champness, 1971).

The perceptual and cognitive processes involved in gaze perception have been traditionally studied in laboratories with carefully controlled stimuli and viewing conditions, such as static images of faces presented on a computer screen. Even though this approach has its undisputed merits, i.e., the controllability and the possibility to isolate the essential features of stimuli for social perception, this approach suffers from obvious shortcomings in ecological validity. In many studies, the images do not even depict real human faces, but very simple schematic drawings of faces. Recently, several researchers in the field of social cognition and social neuroscience have criticized 
Running head: MENTALIZING EYE CONTACT

this approach (Kingstone, Smilek, \& Eastwood, 2009; Risko, Laidlaw, Freeth, Foulsham, \& Kingstone, 2012; Schilbach et al., 2013; Teufel, Alexis, Clayton, \& Davis, 2010; Hari \& Kujala 2009, Hietanen et al., 2008). In studies investigating the effects of direct gaze (eye contact), for example, the use of images versus live persons as stimuli has potentially a substantial influence on the observed responses. Obviously, passive viewing of an image of a person displaying direct gaze does not require similar reactive responses as seeing a person gazing oneself in a live social interaction. Facing a live person with direct gaze involves being the actual object of the other person's attention. In this situation, direct gaze is a strong signal of a possible initiation of interaction. Facing a live person involves 'online' mentalizing consisting of constant implicit prediction and comparison processes characteristic to active interaction, as opposed to 'offline' mentalizing, i.e., passive viewing of such an interaction (cf., Schilbach, 2014). Indeed, previous research has shown that direct gaze is accompanied with increased autonomic nervous system activation and brain activity associated with approach motivation when facing a real person, but not when viewing an image (e.g., Hietanen et al. 2008; Pönkänen, Peltola, \& Hietanen, 2011). Eye contact with a real person is sufficient for initiation of these autonomic preparatory processes. However, an interesting question is whether these processes could be initiated with voluntarily imagining a social interaction involving eye contact or whether they are specific to online mentalizing.

A probable driving force for the differential responses to eye contact between a live gaze and a pictorial gaze stimulus is the participant's experience of being the object of another person's attention. In a previous study, the experience of being the object of the other person's attention during eye contact was explicitly isolated and manipulated in two conditions (Myllyneva \& Hietanen, 2015a). The participants were facing a live stimulus person and, in one condition, they believed that the stimulus person was also seeing them, whereas, in the other condition, they believed that the stimulus person could not see them (due to a mock one-way mirror inserted 
Running head: MENTALIZING EYE CONTACT

between them). Enhanced autonomic (skin conductance and heart rate deceleration) and attentionsensitive brain responses (P3 event-related potential) as well as higher levels of self-evaluated public self-awareness and social presence to eye contact versus averted gaze were observed only when the participants believed that they were being seen. Thus, the study provided strong evidence that the essential factor underlying the increased responses to eye contact was the knowledge of being seen by another person. In another study, it was further investigated whether the mere belief of being seen by another person is sufficient to trigger the enhanced response even when the viewer does not see the other person at all, or whether these responses are intimately tied to seeing the other person during the eye contact (Myllyneva \& Hietanen, 2015b). Despite heightened selfawareness - attesting of differences in higher-order cognition - the belief of being seen by itself did not elicit corresponding physiological responses in the absence of any visual information about the other person. This suggests that the eye contact effects are conditional, not only on the observer spontaneously believing to be seen by the other person, but also on that the observer himself/herself sees the other person. Thus, previous research has shown that a top-down manipulation of the participants' beliefs of being seen or not by another person during eye contact has a strong effect on whether the eye contact results in enhanced physiological and subjective responses or not. However, it remains possible that perceiving a person's image and voluntary mentalizing that the person sees oneself could also trigger similar enhanced physiological and subjective responses spontaneous online mentalizing.

In this study, we were interested in investigating whether the participants' voluntary mentalizing of being seen or not by another person is powerful enough to result in modulation of the physiological, behavioural, and subjective responses in a condition when they are not facing another, live person, but a pre-recorded video of a person. We instructed the participants to immerse as vividly as possible in an imagined social interaction with a pre-recorded stimulus person on a video clip and asked them to imagine the video person as a real, living person sitting in front of 
Running head: MENTALIZING EYE CONTACT

them in the same room. For one group of participants, we also asked them to mentalize that the other person was able to see them, whereas the other group of participants was asked to imagine that a half-silvered mirror was placed between them and the other person so that they could see the other person but this person could not see the participant. Thus, the only difference between the conditions was in the participants' attribution of the other person's ability to see oneself. The contrast between the mutual and one-way visibility was created in a comparable way in a previous study (Myllyneva \& Hietanen, 2015a).

In the present study, we collected three types of data: participants' autonomic responses (skin conductance responses and heart rate), looking behaviour (gaze tracking), and subjective evaluations. Previous studies have shown larger skin conductance responses (SCRs) in response to faces with direct versus averted gaze, but only when the faces were presented live and not when presented as pictures (Helminen et al., 2011; Hietanen et al., 2008; Pönkänen, Peltola, \& Hietanen, 2011). Moreover, as cited above, the enhanced SCRs in response to a live face with direct gaze are also conditional to the observer's belief of being seen by the other person (Myllyneva \& Hietanen, 2015a). In the present study, we measured SCRs to investigate whether the voluntary mentalizing of being seen or not is powerful enough to modulate sympathetic affective arousal (Dawson, Schell, \& Filion, 2000) in response to eye contact also when facing a video-clip of a person. In a similar vein, we investigated whether heart rate (HR) deceleration in response to eye contact is modulated by the observer's condition of mentalizing. HR deceleration response is an index of attention orienting to external stimuli (Graham \& Clifton, 1966), amplified by affectively and motivationally salient stimuli (Bradley, 2009; Lang \& Bradley, 2010). Consistent with behavioral studies showing that direct gaze captures and holds visual attention (e.g., Böckler et al., 2014; Conty et al., 2006, Senju \& Hasegawa, 2005; von Grünau \& Anston, 1995), previous studies have shown enhanced HR deceleration responses to direct versus averted gaze (Akechi et al., 2013). However, also this response has been shown to be modulated by the participants' belief of whether the other person can 
Running head: MENTALIZING EYE CONTACT

see or cannot see him/her during eye contact (Myllyneva \& Hietanen, 2015a). In line with the physiological measures, subjective evaluation of social presence is also modulated by the belief of whether another person can see oneself or not (Myllyneva \& Hietanen, 2015a). Therefore, we measured social presence as well as affective arousal and valence also in the present study.

Previous studies using static and dynamic facial stimulus images have indicated that the eye region is fixated longer than other face regions (e.g., Birmingham, Bischof, \& Kingstone, 2008; Foulsham, Walker, \& Kingstone, 2010). Fixation times in the eye region, nevertheless, depend on (eye) gaze direction: direct gaze has been shown to receive fixations with shorter latencies and longer durations (dwell times) than averted gaze both for still facial images and for animated video clips (Palanica \& Itier 2011; Wieser et al., 2009). Importantly, gazing on the eye-region has been found to be further modulated depending on whether the viewers have been looking at live people or people appearing in videos: in case of eye contact, the eye region of a live person was fixated longer than other parts of the stimulus but not so in the case of a pre-recorded video of the person (Freeth, Foulsham, \& Kingstone, 2013). Thus, because previous studies have shown that observers' gazing behaviour on another person's eye region may differ depending on this person's ability to see the observer (i.e., live people vs. videos), we were naturally interested in investigating whether our mental state manipulation would influence the participants' gazing behaviour in this study.

Based on the results from previous experiments, we expected that voluntary mentalizing would have a similar effect as spontaneous mentalizing on the autonomic responses to direct relative to averted gaze: Both SCR and HR deceleration responses to direct gaze would be enhanced only when the participants voluntarily mentalize that the person on the video is able to see them. We also expected that direct gaze would hold overt attention longer compared to averted gaze, and, furthermore, that voluntary mentalizing of being seen, compared to not being seen, by the person on the video would increase this difference in the overall fixation dwell times. 


\section{Materials and Methods}

\section{Participants}

60 adult university students (40 female and 20 male, age range 19-44, $M \pm S D=25.17 \pm$

5.2) participated in the study. A written consent was received from all participants. Ethical statement for the study was obtained from the Tampere Area Ethical Review Board. The sample size was estimated based on the samples and effect sizes of a previous study (Myllyneva \& Hietanen, 2015a). To examine the adequacy of the sample, an a priori type of power analysis for a 2 x 2 mixed design repeated measures ANOVA was conducted after the experiment using G*Power 3.1 software (Faul, Erdfelder, Lang, \& Buchner, 2007), with the parameters of 95\% power, expected effect size of at least $0.1\left(\eta_{p}^{2}\right)$ (.23 in Myllyneva \& Hietanen, 2015a), an alpha level of .05, the default within-subjects measurement correlation of .5 , and a non-sphericity correction value $(\varepsilon)$ of 1 . This calculation suggested a total sample size of 24 participants (12 participants/group).

\section{Stimuli and procedure}

Stimulus videos. Short ( $5 \mathrm{~s}$, the same duration as for stimuli in Myllyneva \& Hietanen, 2015a) video clips of faces of two males and two females pictured against a black background were prepared with the resolution of $1280 \times 1024$ (Fig. 1) and presented with a frame rate of $25 \mathrm{fps}$. In each video clip, to ensure gaze direction specific responses to the stimuli, the gaze of the person was directed throughout the video either to the participant (direct gaze), downward (downcast gaze), or 30 degrees to the left or right (lateral gaze). During preparation of the video clips, the model persons were asked to maintain a neutral expression and, to prevent any unwanted physiological responses to sudden movements from confounding the data, sit as still as possible without any gross facial, head, or body movements, but blinking was allowed. Each participant viewed video clips of one pseudorandomly selected male and one female, on a $1980 \times 1024$ display at a distance of $70 \mathrm{~cm}$, so that the person appeared in the size of a real person at this typical 
Running head: MENTALIZING EYE CONTACT

interpersonal distance in social interaction. Each male and female person featured in an equal number of video clips in each experimental group (see below).

\section{$<$ Insert Figure 1 about here>}

Procedure. Following consenting to participate and attachment of the electrodes, participants were seated in front of a computer in a darkened laboratory room. The participants were randomly assigned to one of two experimental groups (30 participants in each), except for that the gender distribution was balanced between the groups (10 male and 20 female participants in each group). The experimental groups did not differ from each other with respect to participants' age, $t(58)=.74, p=.46$. Participants in both groups were asked to perform a mentalizing task. In both groups, the participants were encouraged to imagine as vividly as possible that there is another person sitting opposite to them, and that they would know and sense that the person can hear and could even touch them. In one group, the participants were instructed to mentalize that the person appearing on video would see the participant just like the participant saw this person (mentalizing of being seen). For the other group, the participants were instead asked to imagine that a one-sided mirror (such as those seen in TV police series) was placed between them and they were asked to mentalize that, therefore, the video person could not see the participant (mentalizing of not being seen). The experimental groups did not differ in any other respect except for this aspect of instructions given to them before the experimental task.

In each gaze direction condition, the stimuli were shown 8 times (for the lateral gaze direction, 4 stimuli with gaze direction to the left and 4 to the right). Participants thus viewed a total of 24 videos. In each gaze condition, a half of the stimuli pictured a male model and the other half a female model. Different gaze directions were presented in pseudorandom order so that the same gaze direction of a given person could not appear more than twice in a row. The inter-stimulus interval was 20 seconds and the total data collection took about 10 minutes for each participant. 
Running head: MENTALIZING EYE CONTACT

Following the experimental procedure, two self-assessment questionnaires were administered to the participants in both groups: the social presence form (SPF) (Short, Williams, \& Christie, 1976), self-assessment SAM manikins measuring the experienced arousal and valence (Bradley \& Lang, 1994). The participants in each group were asked to evaluate these dimensions of their subjective experience during the experimental task on 7-point scales. Finally, the participants were briefly debriefed. All participants reported having no trouble in following the instructions for the experimental task.

\section{Data acquisition}

Acquisition of the physiological data. Skin conductance measurements were carried out by attaching two $\mathrm{Ag} / \mathrm{AgCl}$ electrodes filled with isotonic paste to the palmar surface of distal phalanges of the participant's left hand's index and middle fingers. HR was measured with two electrodes $(\mathrm{Ag} / \mathrm{AgCl})$ that were applied with electro-conductive paste and placed on both arms. Both signals were sampled and digitized at the rate of $1000 \mathrm{~Hz}$ with a quickAmp amplifier (Brain products GmbH, Munich, Germany). Room temperature during the SCR measurements varied between 19.8 and $23.7 \mathrm{C}^{\circ}(M \pm S D=22.15 \pm .84)$. The experimental groups did not differ from each other with respect to room temperature during the measurement, $t(58)=1.02, \mathrm{p}=.31$.

Eye movements. Binocular eye position was monitored using an eye tracker glasses (SMI ETG) with a sampling rate of $30 \mathrm{~Hz}$. The glasses had a built-in scene camera, recording an area of a viewer's visual field of about $50^{\circ}$ (horizontal) $\times 40^{\circ}$ (vertical) with the point of regard superimposed over the image as a circular cursor in the recorded video. The recorded video allowed a frame-byframe analyses of the gaze direction with a spatial resolution of approximately $0.1^{\circ}$ with typical gaze position accuracy of $0.5^{\circ}-1^{\circ}$. For the eye tracker calibration, participants successively fixated on four points on the display before the experimental task. Offline recalibration based on eye ball analysis of the eye tracking data was performed if substantial drift occurred during the experimental 
Running head: MENTALIZING EYE CONTACT

session. The low sampling frequency and spatial resolution only allowed a long-term measure such as an overall fixation dwell time in the eye region to be meaningfully extracted from the data.

\section{Data analysis}

SCR. The SCR data were re-sampled offline to $100 \mathrm{~Hz}$ and filtered with a $10-\mathrm{Hz}$ low-pass

filter. The skin conductance response was defined as a maximum amplitude change from the baseline level (at the stimulus onset) during a 5-s time period starting $1 \mathrm{~s}$ after the stimulus onset. In case there was more than a $0.1 \mu \mathrm{S}$ amplitude rise during the first second after stimulus onset, the trial was rejected. Of all trials $7.7 \%$ were eliminated due to this criterion or because of a technical error. The data were averaged for each condition and gaze direction for each participant, including those with a maximum amplitude below $0.01 \mu \mathrm{S}$ (i.e. zero responses); this calculation results in the magnitude of the skin conductance responses; a measure that combines response size and response frequency (cf., Dawson et al., 2000).

The SCR data were not normally distributed, and this was corrected for by applying a lntransformation. The width of the inter-quartile range (wIQR) was calculated for each gaze direction. Data of those participants whose responses were three wIQRs further from either edge of the IQR were identified as outliers and excluded from further analyses (5 participants). All analyses were conducted with normally distributed transformed values. Nevertheless, the untransformed values were used to describe the mean values.

HR. The ECG data were prepared offline for the analysis by measuring the time intervals between two successive R-waves (inter-beat interval, IBI) using an in-house (Matlab-based) algorithm. In this phase, trials with excessive signal distortion were removed from the data before further analyses (less than 1\%). Data from three participants in the imagined mutual visibility group were rejected from further analyses because of poor data quality. 
Running head: MENTALIZING EYE CONTACT

The IBIs were quantified and assigned to 1-s intervals for a 9-s period including $3 \mathrm{~s}$ prestimulus within each trial. IBIs were then converted to beats per minute (bpm) and averaged across trials for each condition. The average of the bpms during the pre-stimulus period served as a baseline, from which the bpm of each post-stimulus 1-s interval was subtracted, yielding the HRchange scores with which the analyses were performed.

Gaze tracking. AOIs for the eye region were defined for each video person to measure fixation dwell times to the eye region in each condition. The eye region was defined as covering horizontally an area between the left and right outline of the face and vertically an area between the inner (lower) edges of the eyebrows and the halfway of the nasal bridge (see Fig. 1). The area was individually adjusted for each stimulus person but held similar across all gaze directions. The dwell time was calculated as the total sum of fixation times inside the AOI across all trials in each gaze condition. The dwell times can thus be viewed as 'macro-fixations', organizing all individual fixations into one large-scale scale measure (Salvucci \& Goldberg, 2000).

Data from two participants were rejected due to excessively poor data quality, leaving gaze tracking data of 29 participants from each experimental group for the analyses.

The data from gaze tracking and autonomic measures were analyzed by conducting a mixed design $3 \times 2$ repeated measures analyses of variance (ANOVA) with a within-subjects factor of Gaze direction (direct, lateral, downcast), and a between-subjects factor of Condition of mentalizing (being seen, not being seen). For the analysis of the HR-change scores, a withinsubjects factor Time interval was added (time interval after the stimulus onset: $0-1 \mathrm{~s}, 1-2 \mathrm{~s}, \ldots, 5-6$ s). Greenhouse-Geisser correction procedures were applied when appropriate. 


\section{Results}

Questionnaires. The experimental groups did not differ significantly from each other in self-reported social presence $\left(M_{\text {being seen }}=23.37, S D=5.93 ; M_{\text {not being seen }}=22.80, S D=5.08\right), t_{(58)}=$ $.40, p=.69, d=.11$. The subjective arousal $\left(M_{\text {being seen }}=3.73, S D=1.55 ; M_{\text {not being seen }}=3.77, S D=\right.$ $1.41), t_{(58)}=.08, p=.93, d=.03$, and valence ratings $\left(M_{\text {being seen }}=5.93, S D=1.51 ; M_{\text {not being seen }}=\right.$ $5.43, S D=1.25), t_{(58)}=1.38, p=.17, d=.36$, were not significantly different between the groups, either.

Physiological responses. There was no significant difference in the SCRs with respect to Gaze direction, $\left(M_{\text {direct gaze }}=.064 \mu \mathrm{S}, S D=.06 ; M_{\text {lateral gaze }}=.059 \mu \mathrm{S}, S D=.10 ; M_{\text {downcast gaze }}=.053\right.$ $\mu \mathrm{S}, S D=.07), F_{(2,106)}=.68, p=.508, \eta_{p}^{2}=.013$, or Condition of mentalizing $\left(M_{\text {being seen }}=.059 \mu \mathrm{S}\right.$, $\left.S D=.06 ; M_{\text {not being seen }}=.059 \mu \mathrm{S}, S D=.06\right), F_{(1,53)}=.12, p=.733, \eta_{p}^{2}=.002$. Neither did the Gaze direction $\times$ Condition of mentalizing interaction reach significance, $F_{(2,106)}=.83, p=.440, \eta_{p}^{2}=$ .015. (Figure 2a)

For heart rate deceleration responses, the main effects of Condition of mentalizing, $F_{(1,55)}=$ 5.73, $p=.020, \eta_{p}^{2}=.094$, and Time were significant, $F_{(5,275)}=5.70, p<.001, \eta_{p}^{2}=.094$ (Figure $2 \mathrm{~b}$ ). These significant main effects were qualified by the Condition of mentalizing $\times$ Time interaction, $F_{(5,275)}=5.43, p<.001, \eta_{p}^{2}=.090$, indicating that there was a heart rate deceleration response in the mentalizing of being seen condition, but not in the mentalizing of not being seen condition (see Fig. 2b). Point-by-point comparisons between the experimental groups (Condition of mentalizing) showed that heart rate change differences were significant for all 1-s time-intervals between 2 and 6 seconds post-stimulus, all $p s<.03$. However, no main or interaction effects involving the factor of Gaze direction reached significance $(p s>.16)$. 
Running head: MENTALIZING EYE CONTACT

Eye tracking. The gaze direction had an effect on the AOI dwell times, $F_{(2,112)}=19.71$, p < $.001, \eta_{p}^{2}=.260$ (see Figure 2c). Paired comparisons showed that the dwell times were significantly longer for direct gaze $\left(M_{\text {direct gaze }}=26.49 \mathrm{~ms}, S D=9.34\right)$ than lateral gaze $\left(M_{\text {lateral gaze }}=22.43 \mathrm{~s}, S D\right.$ $=8.33), t_{(57)}=4.33, p<.001, d=.459$, and downcast gaze $\left(M_{\text {downcast gaze }}=21.53 \mathrm{~s}, S D=7.76\right), t_{(57)}=$ $1.20, p<.26, d=.578$. There was no significant difference in dwell time between the lateral and downcast gaze, $t_{(56)}=5.97, p<.001, d=.111$. Condition of mentalizing did not significantly modulate the dwell times, $F_{(2,112)}=1.01, \mathrm{p}=.319, \eta_{p}^{2}=.018$, nor was there a significant interaction between Condition of mentalizing and Gaze direction, $F_{(2,112)}=1.20, \mathrm{p}=.306, \eta_{p}^{2}=.021$.

\section{$<$ Insert Figure 2 about here>}

Bayesian statistics. Null hypothesis testing is asymmetric in that it only yields conclusive evidence for rejecting the null hypothesis; a nonsignificant result even with a p-value close to 1 cannot be used as support for the null hypothesis. Symmetric statistical inference approaches such as calculation of Bayes factor (B; see Dienes, 2014) can be used in simultaneous evaluation of both the null and the alternative hypotheses relative to a given data. B reflects the relative likelihood of one hypothesis over another: $\mathrm{B}=1$ represents equal likelihood for both hypotheses, and in the following analysis, Bs $>1$ indicate greater relative likelihood for the null hypothesis and $\mathrm{Bs}<1$ for the alternative hypothesis. In other words, B constitutes a continuous index of toward which hypothesis (and how far) to revise the interpretation in light of the data.

Following the approach by Dienes (2014), we tested further the non-significant Gaze direction $\times$ Condition of mentalizing interactions obtained for skin conductance and heart rate responses by calculating Bs. We reasoned that the interaction raw effects (difference between the mentalizing conditions [mutual visibility - one-way visibility] in the direct gaze minus averted gaze mean SCR/HR response values) could be uniformly anything between zero and those observed in Myllyneva \& Hietanen (2014a, Exp. 1; SCR: .023 $\mu$ S; HR: 1.36 bpm). Given the corresponding 
Running head: MENTALIZING EYE CONTACT

interaction raw effects observed in the present data (SCR: -.006 $\mu \mathrm{S}$; HR: -.14 bpm), the null hypothesis of no interaction raw effect (zero), and the alternative hypotheses of any effect with a value between zero and the theoretically maximum interaction raw effects, resulted in $B_{S C R}=2.78$ and $B_{H R}=1.86$.

\section{Discussion}

In this study, we investigated whether people's voluntary mentalizing of being seen or not by another person is powerful enough to result in modulation of physiological and gaze tracking responses to eye contact also when they are not facing another, live person, but a pre-recorded video of a person. In previous studies, greater autonomic responses (skin conductance and heart rate deceleration) to eye contact versus averted gaze were observed only if the participants saw another, live person (Hietanen et al., 2008) and when seeing a live person, if they believed they were seen by that person, (Myllyneva \& Hietanen, 2015a). The present results showed, however, that the gaze direction of a person appearing on a video did not have an effect on skin conductance or heart rate deceleration responses, no matter whether the participant voluntarily mentalized that the person was able to see them or not. This result seems to corroborate the idea that seeing another, live person and the belief of being seen by that person are essential components for enhanced autonomic responses to eye contact. The present results suggest that the implicit mentalizing processes, which are triggered by seeing a live person with direct gaze and which are likely to be necessary for enhanced autonomic responses, are context-sensitive and spontaneous, and cannot be reproduced under voluntary cognitive control.

Previous studies by Teufel and colleagues showed that visual attention orienting by gaze direction cues (Teufel et al., 2010) and sensory visual adaptation to gaze direction stimuli (Teufel et al., 2009) were modulated by the belief of whether the person appearing on a video could or could not see the participant. However, there is one critical difference between the present study and those 
Running head: MENTALIZING EYE CONTACT

by Teufel and colleagues. In Teufel et al. studies the participants were deceived to believe that there was a bi-directional, on-line videolink between the participant's room and the adjacent room where the other person was supposed to sit. Thus, the manipulation of the mental state attributions seems to be effective only if participants believe that they are truly interacting with a live person (even if this person only appears on a video).

Regarding the total lack of gaze direction effect on autonomic responses, the present results are compatible with previous studies showing that, in contrast to gaze direction of a live person, the gaze direction of a static facial image presented on a computer monitor did not have an effect on autonomic or brain responses (Hietanen et al. 2008; Pönkänen, Alhoniemi, Leppänen, \& Hietanen, 2011; Pönkänen, Peltola, \& Hietanen, 2011). Thus, it seems that the lack of gaze direction effect by face images in previous studies was not due to the fact that, unlike a face of a live person, the static face images were devoid of any kinematic information. Instead, together with previous results, the present results provide further support to our suggestions that the lack of gaze direction effects on autonomic responses by facial images, whether static pictures or dynamic video clips, is related to the fact that a person appearing in an image does not look back to the observer; the self is not seen by another mind (Hietanen et al., 2008; Pönkänen, Alhoniemi, Leppänen, \& Hietanen, 2011; Pönkänen, Peltola, \& Hietanen, 2011).

The condition of mentalizing, however, did exert an influence on HR deceleration: regardless of gaze direction, HR deceleration, a physiological marker related to attention orienting, was greater in the 'being seen' than in the 'not being seen' mentalizing condition. This result seems to suggest that the participants were, after all, able to voluntarily mentalize being seen or not by a person appearing on a video and that, in the 'being seen' condition, the presentation of the stimulus person resulted in enhanced orienting of attention. However, drawing this conclusion may not be warranted, especially because the self-evaluations and the skin conductance responses did not show the same pattern. It is also possible that the 'not being seen' condition was cognitively more 
Running head: MENTALIZING EYE CONTACT

demanding than the 'being seen' condition. The 'not being seen' condition required imagination of the interaction plus the presence of the one-way window preventing the stimulus person from seeing the self. Thus, it required intensive allocation of attention and, therefore, the attention orienting triggered by the external stimulus was attenuated in this condition. This speculation is supported by the finding that, in the 'not being seen' condition, there actually seemed to be a slight HR acceleration response indicative of sympathetic nervous system activation. Such a response might stem from increased cognitive effort, which has been associated with a HR acceleration response (e.g., Kennedy \& Scholey, 2000).

Interestingly, stimulus gaze direction had an effect on participants' own gaze behavior: the eye region of faces with direct gaze was viewed longer than the eye region of faces with averted gaze. This finding is in line with results from previous studies showing that the eye regions of virtual characters, real persons appearing in still and video images as well as that of live stimulus persons were fixated longer when the character/person was in eye contact compared to when looking away (Palanica \& Itier, 2006; Wieser et al., 2009; Freeth et al., 2013). Gaze tracking data did not show, however, any effects by the mentalizing manipulation. Results from one previous study showed increased viewing of eye region in eye contact vs. looking away for live stimulus persons relative to video-transmitted persons (Freeth, et al., 2013). Thus, compatible with the reasoning presented above, mentalizing of being seen by a person appearing in a video does not enhance viewing of the stimulus person's eye region similarly to when facing another person live.

Complying with the results from the measures of autonomic arousal, the subjective measures of social presence and affective arousal and valence did not differ between the mentalizing conditions. Compared with results from previous studies showing an increase in subjective measures when believing being seen vs. not being seen by another live person (Myllyneva \& Hietanen, 2015a, 2015b), this result adds up to the idea that the same mentalizing processes are not initiated in eye contact with a person appearing in a video as compared to eye contact with a live 
Running head: MENTALIZING EYE CONTACT

person. It seems that the autonomic and subjective responses to eye contact depend on appropriate mentalizing processes - those reflecting the automatic contextual knowledge about being seen by another person.

An important issue for consideration is, of course, whether the lack of greater autonomic and subjective responses to eye contact in the 'being seen' vs. 'not being seen' condition reflected a lack of participants' capability of voluntarily imagining being in interaction with a person appearing in a video, or whether it reflected the fact that voluntary mentalizing is not able to trigger the same processes in response to eye contact as what spontaneous mentalizing is capable of. The experimental task seemed easy for the participants, as none of them reported of difficulties in completing the imagination task in debriefing, and we would have excluded data from any such participant reporting difficulties in the task from the analyses. For this reason, and also because we found a difference in the HR response between the conditions (even if it just reflected differential cognitive demands), we believe that the observed results could not have reflected a failure in the imagination task or in the experimental manipulation, but the fact that the voluntary mentalizing is not sufficient to initiate the autonomic responses specific to genuine eye contact. Also, the sample size $(n=60)$ should have been sufficient for observing even subtle differences in autonomic arousal $\left(\eta_{p}^{2}=.1\right.$ vs. $\eta_{p}^{2}=.23$ in Myllyneva \& Hietanen, 2015a), as suggested by the power analysis. Bayesian statistical analyses indicated that support for the absence of genuine eye contact effects on autonomic responses is from $1.86(\mathrm{HR})$ to 2.78 (SCR) times stronger than support for their existence. However, as conventionally $B s>3$ are taken to indicate substantial support for the null hypothesis (Dienes 2014), the data are not quite conclusive.

In conclusion, our findings revealed that imagining eye contact with a pre-recorded stimulus person does not lead to similar enhancement of physiological and subjective responses as eye contact with a living stimulus person. The measured eye contact effects thus seem to depend on 
Running head: MENTALIZING EYE CONTACT

possibility for interaction with a live person. This seems to pose an important challenge for information and communication technology mediated social interaction, and further points to the need to study eye contact effects as well as many other processes of social cognition in natural, social circumstances, instead of using computerized stimuli in impoverished laboratory settings.

\section{Acknowledgments}

This work was supported by the Academy of Finland (MIND program grant \#266187) to JKH. The authors want to thank Jonne Hietanen and Aleksi Syrjämäki for assistance in data collection and preparation. 
Running head: MENTALIZING EYE CONTACT

\section{References}

Akechi, H., Senju, A., Uibo, H., Kikuchi, Y., Hasegawa, T., \& Hietanen, J. K. (2013) Attention to eye contact in the West and East: Autonomic responses and evaluative ratings. PLoS One, 8, e59312.

Birmingham, E., Bischof, W. F., \& Kingstone, A. (2008) Gaze selection in complex social scenes. Visual Cognition, 16, 341-55.

Böckler, A., van der Wel, R. P. R. D., \& Welsh, T. N. (2014) Catching eyes: Effects of social and nonsocial cues on attention capture. Psychological Science, 25, 720-727.

Bradley, M. M. (2009) Natural selective attention: Orienting and emotion. Psychophysiology, 46, $1-11$.

Bradley, M. M., Lang, P. J. (1994) Measuring emotion: The self-assessment manikin and the semantic differential. Journal of Behavior Therapy and Experimental Psychiatry, 25, 49-59.

Conty, L., Tijus, C., Hugueville, L., Coelho, E., \& George, N. (2006) Searching for asymmetries in the detection of gaze contact versus averted gaze under different head views: a behavioural study. Spatial Vision, 19, 529-45.

Cooper, R. M., Law, A. S., \& Langton, S. R. H. (2013) Looking back at the stare-in-the-crowd effect: Staring eyes do not capture attention in visual search. Journal of Vision, 13, 1-22.

Dawson, M. E., Schell, A. M., \& Filion, D. L. (2000) The electrodermal system. In: J. T. Cacioppo, L. G. Tassinary, G. G. Berntson (eds.), Handbook of psychophysiology (2nd ed.), Cambridge University Press. p. 200-23.

Doi, H., Ueda, K., \& Shinohara, K. (2009) Neural correlates of the stare-in-the-crowd effect. Neuropsychologia, 47, 1053-60. 
Running head: MENTALIZING EYE CONTACT

Faul, F., Erdfelder, E., Lang, A., \& Buchner, A. (2007). G*Power 3: A flexible statistical power analysis program for the social, behavioral, and biomedical sciences. Behavior Research Methods, 39(2), 175-191. DOI: 10.3758/BF03193146

Foulsham, T., Walker, E., \& Kingstone, A. (2011) The where, what and when of gaze allocation in the lab and the natural environment. Vision Research, 51, 1920-31.

Freeth M, Foulsham T, Kingstone A (2013) What Affects Social Attention? Social Presence, Eye Contact and Autistic Traits. PLoS ONE 8, e53286.

Graham, F. K., \& Clifton, R. K. (1966) Heart-rate change as a component of the orienting response. Psychological Bulletin, 65, 305-20.

Helminen, T. M., Kaasinen, S. M., \& Hietanen, J. K. (2011) Eye contact and arousal: the effects of stimulus duration. Biological Psychology, 88, 124-30.

Hietanen, J. K., Leppänen, J. M., Peltola, M. J., Linna-aho, K., \& Ruuhiala, H. J. (2008) Seeing direct and averted gaze activates the approach-avoidance motivational brain systems. Neuropsychologia, 46, 2423-30.

Kennedy, D. O., \& Scholey, A. B. (2000) Glucose administration, heart rate and cognitive performance: effects of increasing mental effort. Psychopharmacology, 149, 63-71.

Kingstone, A., Smilek, D., \& Eastwood, J. D. (2008) Cognitive ethology: A new approach for studying human cognition. British Journal of Psychology, 99, 317-45.

Lang, P. J., \& Bradley, M. M. (2010) Emotion and the motivational brain. Biological Psychology, $84,437-50$. 
Running head: MENTALIZING EYE CONTACT

Lyyra, P., Astikainen, P., \& Hietanen, J. K. (2017). Look at them and they will notice you:

Distractor-independent attentional capture by direct gaze in change blindness. Visual Cognition.

Nichols, K. A., \& Champness, B. G. (1971) Eye gaze and the GSR. Journal of Experimental Social Psychology, 7, 623-6.

Myllyneva, A., \& Hietanen, J. K. (2015a) There is more to eye contact than meets the eye. Cognition, 134, 100-9.

Myllyneva, A., \& Hietanen, J. K. (2015b) The dual nature of eye contact: to see and to be seen. Social Cognitive and Affective Neuroscience, 1, 7. DOI: 10.1093/scan/nsv075

Palanica, A., \& Itier, R. J. (2012) Attention capture by direct gaze is robust to context and task demands. Journal of Nonverbal Behavior, 36, 123-34.

Pönkänen, L. M., Alhoniemi, A., Leppänen, J. M., \& Hietanen, J. K. (2011) Does it make a difference if I have an eye contact with you or with your picture? An ERP study. Social Cognitive and Affective Neuroscience, 6, 486-94.

Pönkänen L. M., Peltola M. J., \& Hietanen J. K. (2011) The observer observed: Frontal EEG asymmetry and autonomic responses differentiate between another person's direct and averted gaze when the face is seen live. International Journal of Psychophysiology, 82, 180-187.

Risko, E.F., Laidlaw, K.E.W., Freeth, M., Foulsham, T., \& Kingstone, A. (2012) Social attention with real versus reel stimuli: Toward an empirical approach to concerns about ecological validity. Frontiers in Human Neuroscience, 106, 1487-96 
Running head: MENTALIZING EYE CONTACT

Salvucci, D. D., \& Goldberg, J. H. (2000) Identifying fixations and saccades in eye-tracking protocols. In: Proceedings of the Eye Tracking Research and Applications Symposium. New York: ACM Press. p. 71-8.

Schilbach, L. (2014). On the relationship of online and offline social cognition. Frontiers in Human Neuroscience, 8, 278.

Schilbach, L., Timmermans, B., Reddy, V., Costall, A., Bente, G., \& Schlicht, T. (2013) Toward a second-person neuroscience. Behavioral and Brain Sciences, 36, 393-414.

Senju, A., \& Hasegawa, T. (2005) Direct gaze captures visuospatial attention. Visual Cognition, 12, 127-44.

Senju, A., \& Johnson, M. H. (2009) The eye contact effect: Mechanisms and development. Trends in Cognitive Sciences, 13, 127-34.

Short, J., Williams, E., Christie, B. (1976) The social psychology of telecommunications. New York: Wiley.

Teufel, C., Alexis, D.M., Clayton, N.S., \& Davis, G. (2010) Mental-state attribution drives rapid, reflexive gaze following. Attention, Perception, \& Psychophysics, 72, 695-705.

Teufel, C., Alexis, D. M., Todd, H., Lawrance-Owen, A. J., Clayton, N. S., \& Davis, G. (2009) Social cognition modulates the sensory coding of observed gaze direction. Current Biology, 19, 1274-77.

Von Grünau, M., \& Anston, C. (1995) The detection of gaze direction: A stare-in-the-crowd effect. Perception, 24, 1297-313. 
Running head: MENTALIZING EYE CONTACT

Wieser, M. J., Pauli, P., Alpers, G. W., \& Mühlberger, A. (2009) Is eye to eye contact really threatening and avoided in social anxiety? An eye-tracking and psychophysiology study. Journal of Anxiety Disorders, 23, 93-103. 
Running head: MENTALIZING EYE CONTACT

Figure legends

Fig. 1. Still shots of the video clips depicting the stimulus persons and illustrations (framed by rectangles) of the AOIs used in the gaze tracking analyses. Upper left: direct gaze; upper right: averted gaze to the right; lower left: averted gaze to the left; lower right: downcast gaze.

Fig. 2. a) SCRs, b) Heart rate changes, and c) Fixation dwell times in the eye region for each gaze directions in Mentalizing of being seen and Mentalizing of not being seen conditions. The error bars indicate standard errors of the means. 
Running head: MENTALIZING EYE CONTACT

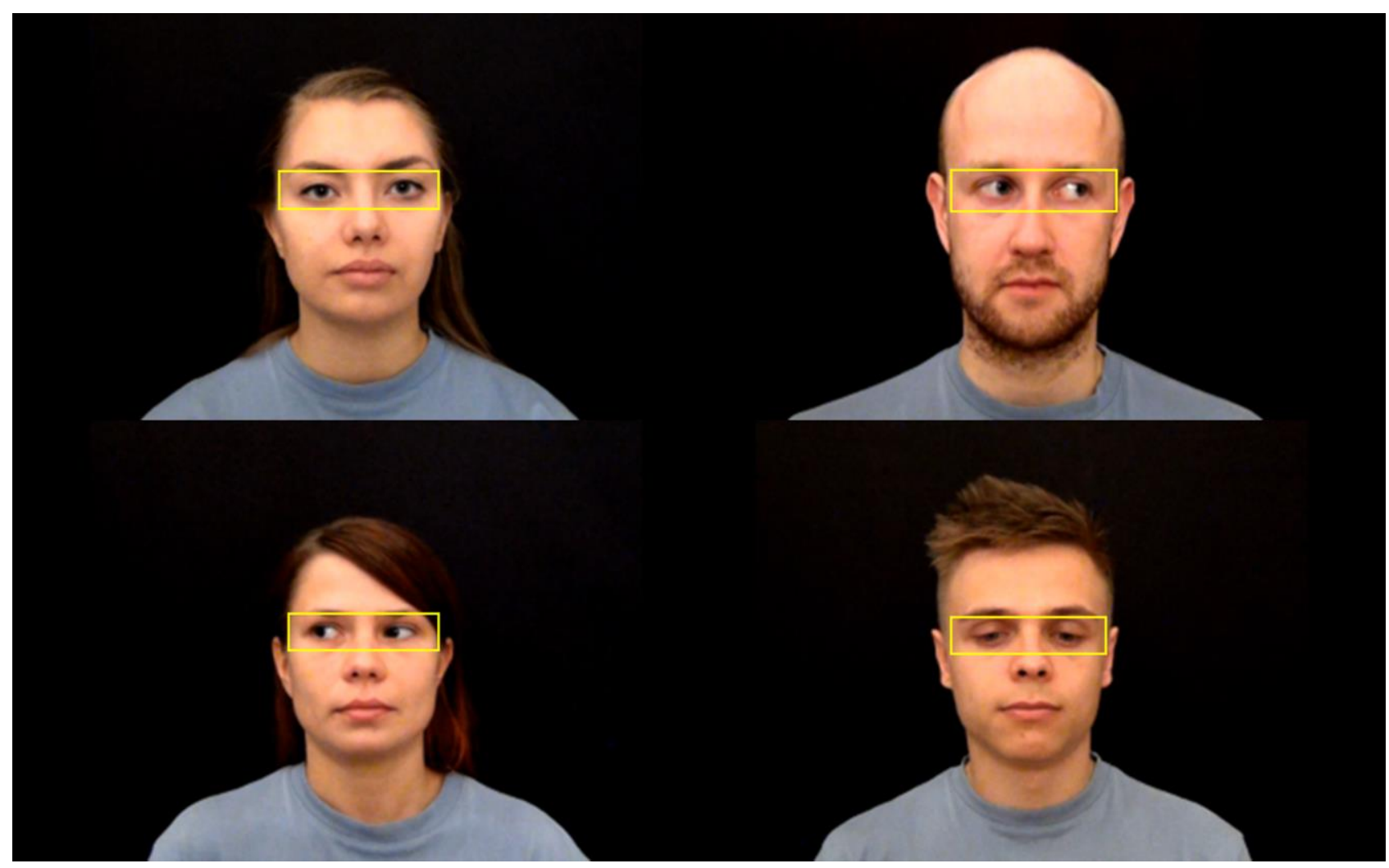

Figure 1. 
Running head: MENTALIZING EYE CONTACT

a)

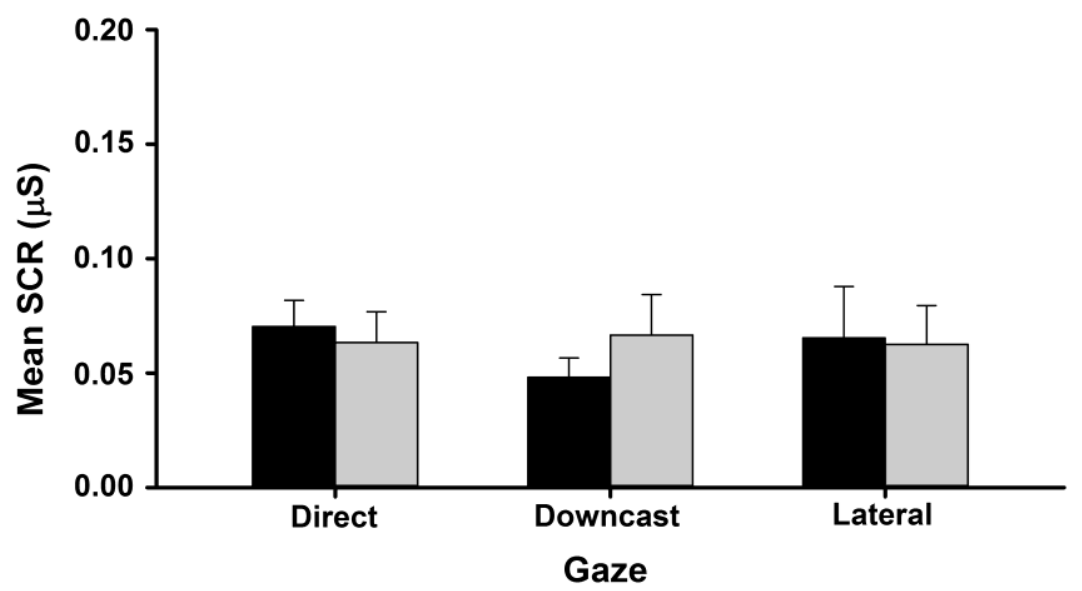

b)

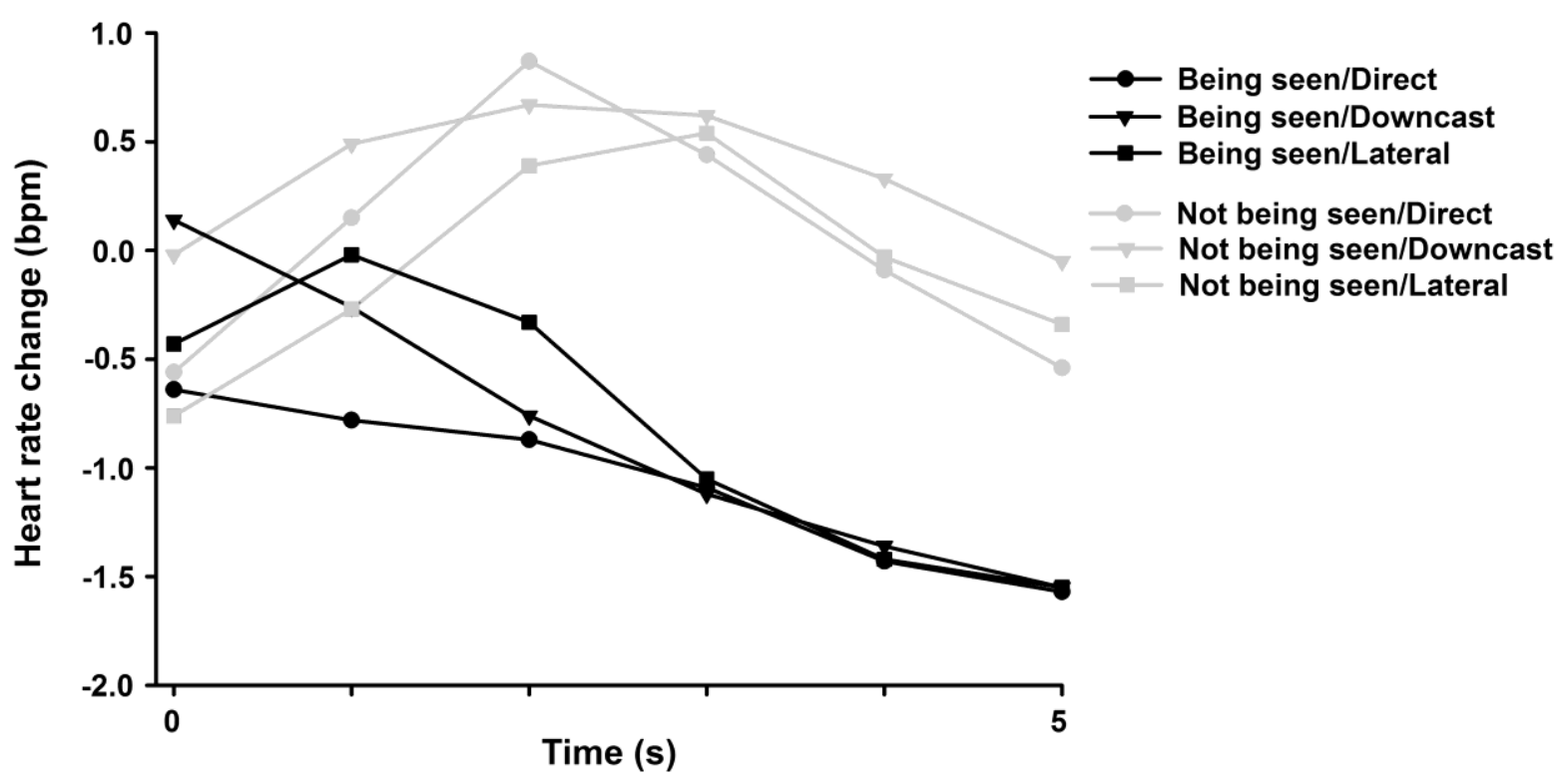

c)

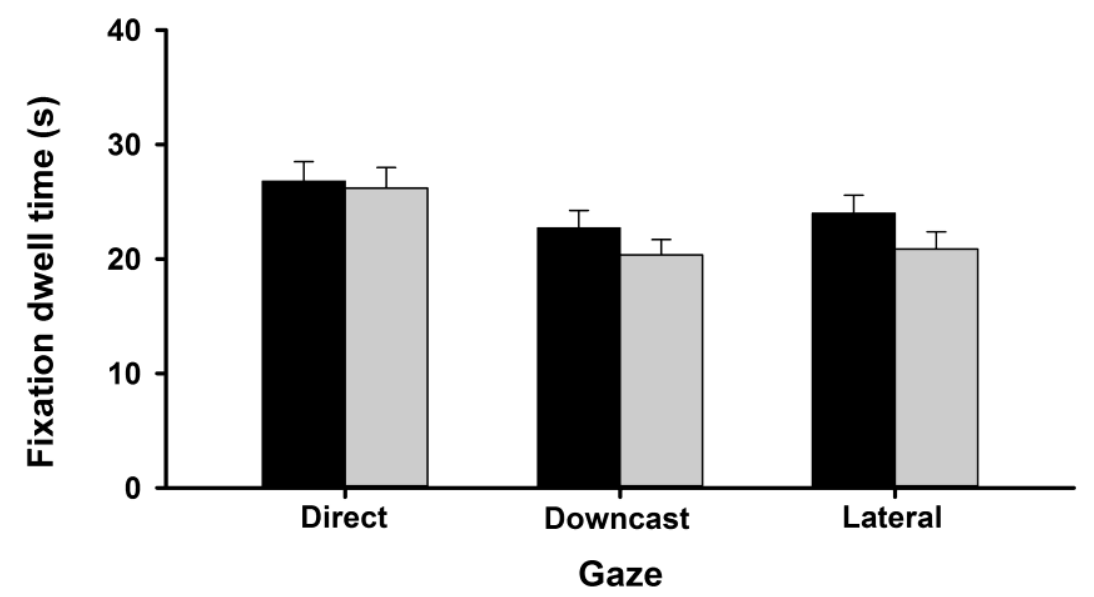

